\title{
Observation of high-energy cosmic rays by very inclined muon bundles in the NEVOD-DECOR experiment
}

\author{
O. Saavedra ${ }^{1, \text { a }}$, N.S. Barbashina ${ }^{2}$, A.G. Bogdanov ${ }^{2}$, D.V. Chernov ${ }^{2}$, L.I. Dushkin ${ }^{2}$, S.S. Khokhlov ${ }^{2}$, V.A. Khomyakov ${ }^{2}$, \\ V.V. Kindin ${ }^{2}$, R.P. Kokoulin ${ }^{2 b}$, K.G. Kompaniets ${ }^{2}$, G. Mannocchi ${ }^{3}$, A.A. Petrukhin ${ }^{2}$, V.V. Shutenko ${ }^{2}$, G. Trinchero ${ }^{3}$, \\ I.I. Yashin ${ }^{2}$, K.O. Yurin ${ }^{2}$, and E.A. Zadeba ${ }^{2}$ \\ 1 Dipartimento di Fisica dell' Universita di Torino, Italy \\ 2 National Research Nuclear University MEPhI (Moscow Engineering Physics Institute), Moscow, Russia \\ 3 Osservatorio Astrofisico di Torino - INAF, Torino, Italy
}

\begin{abstract}
The Russian-Italian NEVOD-DECOR experiment on measurements of the local muon density spectra at various zenith angles gave the possibility to obtain important information on the primary cosmic ray flux and interaction characteristics in a wide energy range from $10^{15}$ to more than $10^{18} \mathrm{eV}$. At large zenith angles and high muon densities, a considerable excess of muon bundles has been found in comparison with expectation. In this paper, an update of these investigations is presented and some new results obtained by the collaboration are discussed.
\end{abstract}

\section{Introduction}

The first cooperation agreement between MEPhI and Torino group (Institute of Cosmo-geophysics, Torino, and Torino University) was signed in June 1992. The main purpose of the cooperation was the creation of the coordinate detector DECOR around the Cherenkov water calorimeter NEVOD [1] for investigations of multiparticle events in cosmic rays at large zenith angles. According to the agreement, streamer tube chambers, front-end electronics and other equipment used earlier in the NUSEX experiment [2] in the Mt. Blanc tunnel were delivered to Moscow. The first supermodules of the detector were put into operation in 1998. In 2001, the construction of the DECOR (eight supermodules with total area about $70 \mathrm{~m}^{2}$ ) was completed [3]. Longterm experiments (about 20,000 hours net operation time) with the NEVOD-DECOR complex were conducted in 2002-2007. The main objects of the investigations were the characteristics of multi-muon events (muon bundles) in a wide range of zenith angles, from about $30^{\circ}$ to the horizon. Unique experimental data on the zenith-angular dependence of muon bundle intensity with particle multiplicities from 3 to about 100 were obtained. A novel approach to the analysis of data on muon bundles based on the phenomenology of the local muon density spectra (LMDS) at various zenith angles was developed [4], that allowed to extract information about the primary cosmic ray flux and interaction characteristics in a record wide energy range from $10^{15}$ to more than $10^{18} \mathrm{eV}$. In 2012, after a deep modernization of the measuring systems of the NEVOD calorimeter [5], the experiments with the complex were resumed, and the available experimental statistics of muon bundles

\footnotetext{
a e-mail: saavedra@to.infn.it

b e-mail: rpkokoulin@mephi.ru
}

has been increased nearly twice. In this paper, a brief overview of the earlier DECOR data and some new results of the analysis of the combined experimental statistics are presented.

\section{Muon bundles in the coordinate-tracking detector}

A general layout of the NEVOD-DECOR is shown in Fig. 1. The side part of the coordinate-tracking detector consists of 8 supermodules (SM) and is deployed in the galleries of the NEVOD building, from three sides of the water tank with inner volume $9 \times 9 \times 26 \mathrm{~m}^{3}$ and $0.5 \mathrm{~m}$ thick concrete walls. Two pairs of SMs are located in the opposite short galleries, and four SMs occupy a long gallery. Each SM has an effective area of $8.4 \mathrm{~m}^{2}$ and consists of eight planes of streamer tube chambers with resistive cathode coating hung vertically with $6 \mathrm{~cm}$ spacing between them. The chambers are operated in a limited streamer mode with a continuous flow of $\left(\mathrm{Ar}+\mathrm{CO}_{2}+\right.$ n-pentane) gas mixture. The length of the chambers is $3.5 \mathrm{~m}$, inner tube cross-section is $9 \times 9 \mathrm{~mm}^{2}$. The planes of the chambers are equipped with a two-dimensional system of external readout strips (256 horizontal X- and 256 vertical Y-strips in each plane with 1.0 and $1.2 \mathrm{~cm}$ pitch, respectively). The spatial and angular accuracy of the reconstruction of the tracks crossing the SM is about $1 \mathrm{~cm}$ and better than $1^{\circ}$, correspondingly. As a first-level trigger condition, a 4-fold coincidence of signals from the planes of the SM is used.

Initially, the coordinate-tracking detector also included four horizontal SMs deployed on movable platforms on the roof of the NEVOD water tank (see Fig. 1). In 2005, the upper supermodules were re-assembled, equipped with a newly developed fast electronics, and a wideaperture muon hodoscope URAGAN [6] with an area 


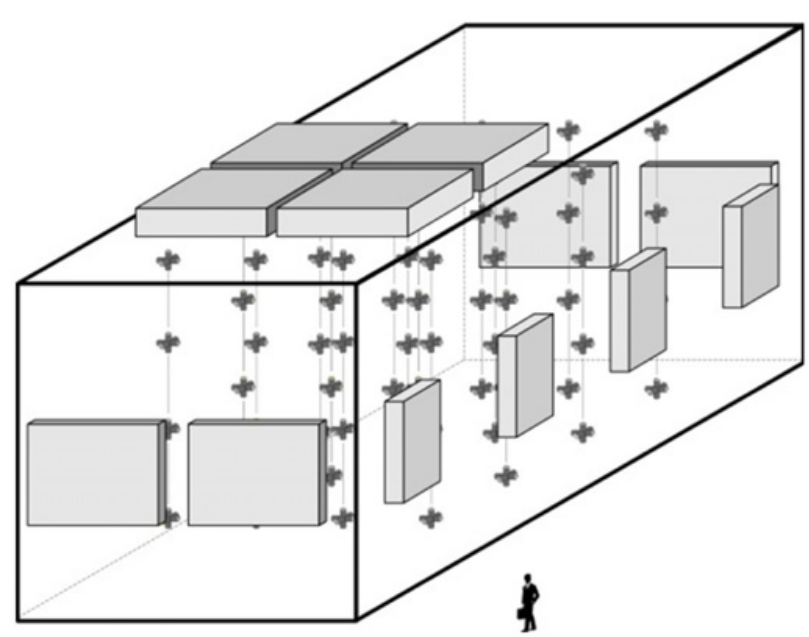

Figure 1. General layout of the NEVOD-DECOR complex.

of about $45 \mathrm{~m}^{2}$ was created on the basis of the upper part of the DECOR. The URAGAN ensures the detection and reconstruction in real-time mode of about 6,000 particle tracks per second, and is now being used for the solution of various tasks related with cosmic ray muon flux variations.

In 2002-2007, a long series of measurements with NEVOD-DECOR complex were conducted. Net operation time with a full configuration of the side DECOR (8 SMs) amounted to $19,922 \mathrm{~h}$. The set of the hardware event selection conditions based on the coincidences of the signals from different parts of the coordinate detector and signals formed by the NEVOD triggering system was kept unchanged during the measurements. Selection of muon bundles from DECOR data is based on the fact that tracks of muons produced in the atmosphere are nearly parallel. The selection procedure included several stages: trigger condition of the coincidence of the signals from at least three DECOR supermodules; off-line track reconstruction and soft program selection of muon bundle candidates containing a required minimal number of quasi-parallel tracks; final event classification and track counting by the operators. Muon bundles leave a very bright signature in the coordinate detector (see Fig. 2), and cannot be mixed with other types of events. A more detailed description of experimental data treatment is given in [7].

Since the procedure of track counting by the operators is rather laborious, the data were analyzed part by part, for different ranges of muon multiplicity and zenith angles. In all, about 40 thousand events were selected from the data accumulated in 2002-2007 measurement series. Phenomenological distributions of muon bundles in particle multiplicity for several zenith angle intervals and in zenith angle for different minimal numbers of muons are presented in Fig. 3. As is seen from the plots, the obtained data on muon bundles cover about six orders of magnitude in the event intensity.

\section{Local muon density spectra at various zenith angles}

At large zenith angles, EAS are detected at the ground as a practically pure muon component. Due to a large distance from the shower generation point to observation level, transverse dimensions of EAS in muons rapidly increase with zenith angle, the effect being additionally enhanced by the particle deflection in the geomagnetic field. Therefore, a muon detector with sizes of the order of tens meters may be considered as a point-like probe. In an individual event, the local density of EAS muons at the observation point may be estimated as the ratio of muon bundle multiplicity to the detector area for a given direction.

Contribution to the events with a certain muon density give showers with different primary energies, detected at different (random) distances from the axis. However, due to a fast decrease of the primary cosmic ray (PCR) intensity with the increase of energy, the effective primary energy band appears relatively narrow (see Fig. 4). It is important to mark that at different zenith angles the events with a fixed muon density are formed by primary particles with substantially different energies. At that, the event collection area is determined not by the detector size, but by the dimensions of the EAS in the muon component which near the horizon reach several kilometers. Two last circumstances allow one to explore a very wide range of PCR energies in a single experiment with a relatively small detector by means of measuring LMDS at various zenith angles.

A general approach to the analysis of DECOR data on muon bundles included the following steps: iterative reconstruction of local muon density spectra in a detector-independent form on the basis of the measured distributions of muon bundle characteristics, taking into account Poisson fluctuations of the number of muons that hit the setup, triggering and selection conditions, detector efficiencies, etc.; calculation of the expected LMDS for various assumptions about the primary spectrum, mass composition and hadron interaction models using two-dimensional muon lateral distribution functions (LDF) simulated by means of the CORSIKA code [8]; comparison of data with calculation results.

In Fig. 5, experimental LMDS reconstructed from DECOR data are compared with CORSIKA-based calculations for 4 different zenith angles $\left(35^{\circ}, 50^{\circ}, 65^{\circ}\right.$, and $78^{\circ}$ ). As a model of the primary flux for the comparison of data and calculations, a power type energy spectrum with differential slope $(\gamma+1)=2.7$ below the knee energy $(4 \mathrm{PeV})$ steepening to $(\gamma+1)=3.1$ above the knee was assumed; absolute normalization was chosen according to the bulk of experimental data around the knee presented in the review [9]; two limiting cases of the mass composition (only protons and only iron nuclei) were considered. The curves in the figure correspond to two widely used hadron interaction models SIBYLL2.1 and QGSJET01 (dashed and solid curves, respectively). Arrows in the upper part of each frame indicate the values of effective (mean logarithmic) primary particle energies.

As seen from the figures, the experimental LMDS is in reasonable agreement with calculations for moderate zenith angles $\left(\theta=35^{\circ}\right.$, upper left frame in Fig. 5) at energies around the knee, including the absolute normalization. The increase of the LMDS slope related with the knee in the primary spectrum is clearly seen. This result may be considered as an indirect check of 

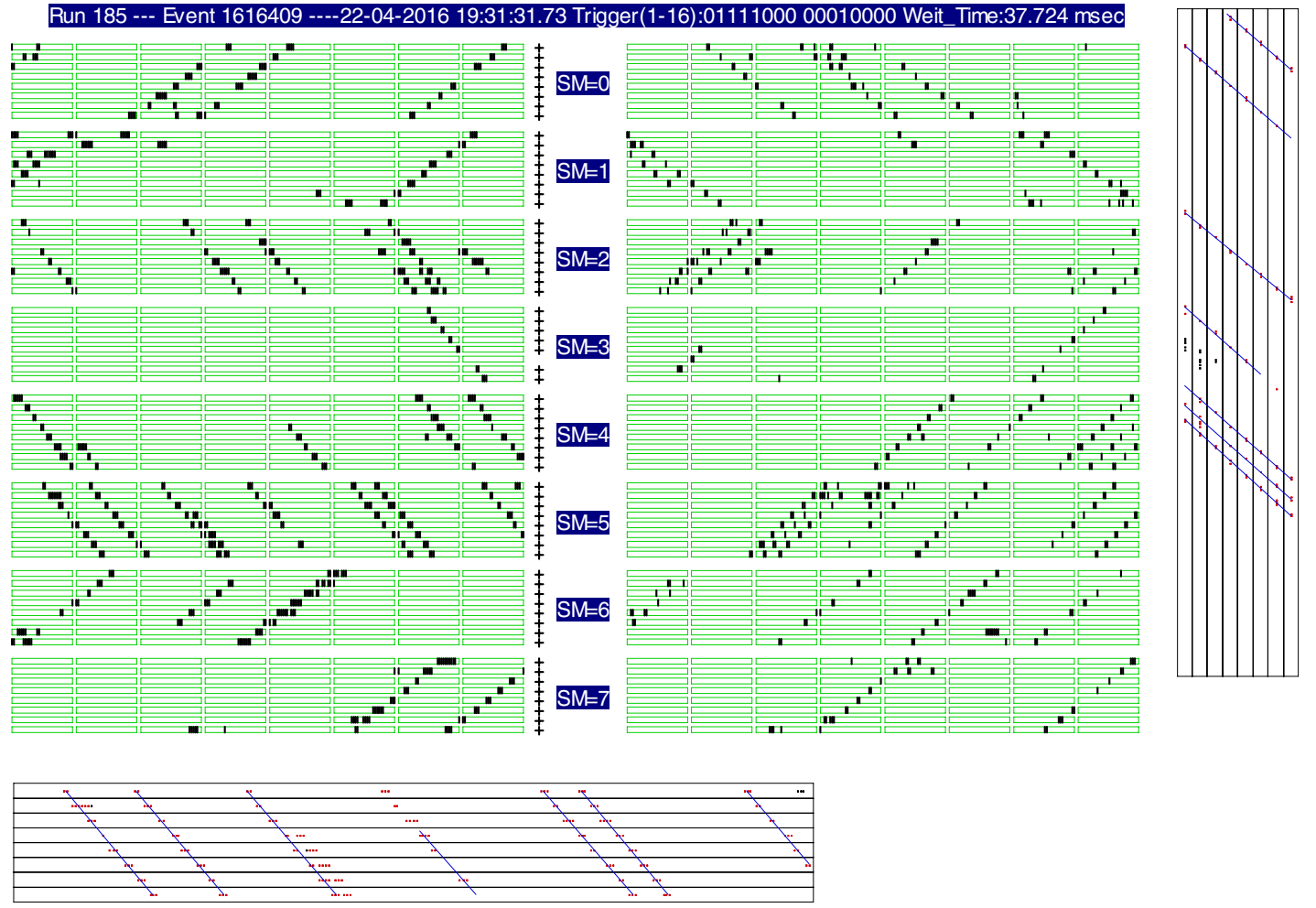

Figure 2. Example of detection of muon bundle event in DECOR. On the right and at the bottom, enlarged views of the response of SM $\sharp 5$ are shown.
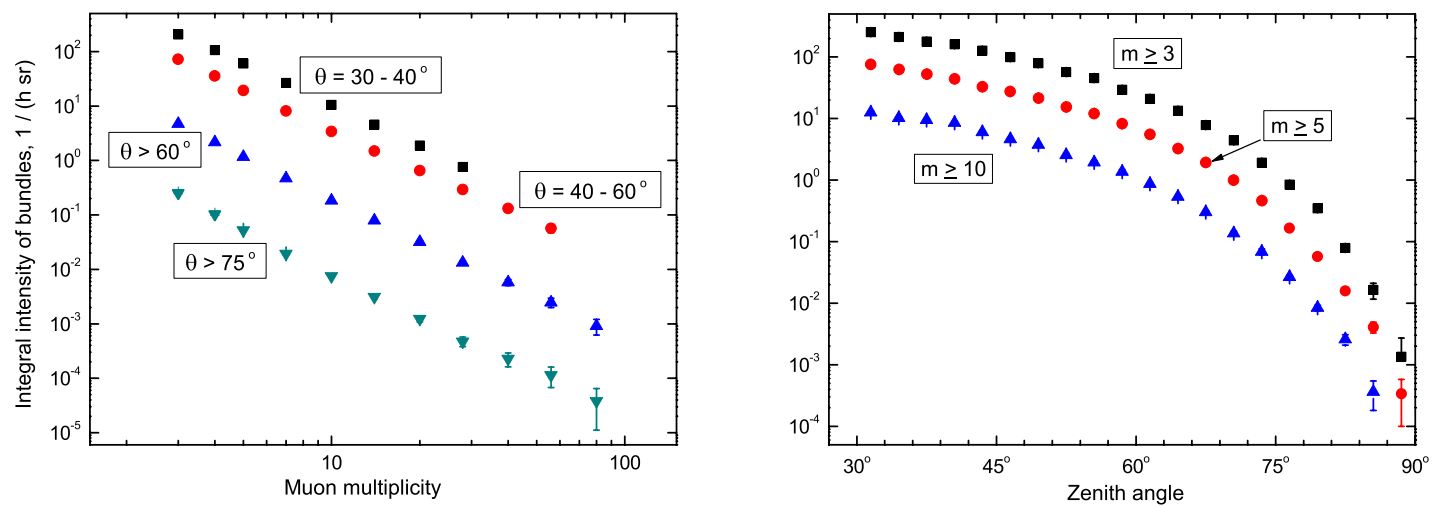

Figure 3. Integral distributions of muon bundles in the particle multiplicity for several ranges of zenith angle (left) and zenith angle distributions of the bundles with different minimal number of muons (right). Data of 2002-2007 [7].

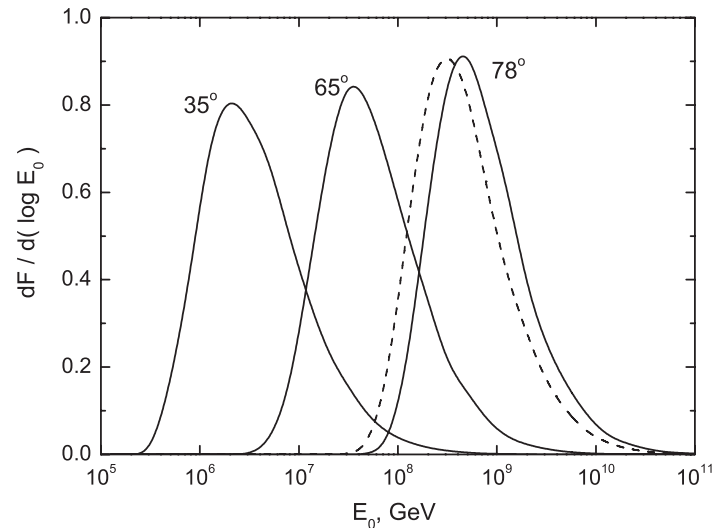

Figure 4. Distribution of primary cosmic ray particle energies contributing to events with a fixed muon density $(D=0.2$ muons $/ \mathrm{m}^{2}$ ) at different zenith angles [7]. Solid curves correspond to calculations for primary protons, dashed curve for iron nuclei. the energy scale calibration. At effective primary energies $E_{0}>10^{16} \mathrm{eV}$ (zenith angles $50^{\circ}$ and $65^{\circ}$ ), a progressive increase of LMDS in comparison with calculations is observed, which can be interpreted as a trend to a heavier mass composition, though an alternative interpretation related with increasing deficit of muons in simulated EAS is also possible. Near $10^{17} \mathrm{eV}$, a hint for a second knee was found: partial fits of the data at $65^{\circ}$ below and above this energy show an increase of the integral LMDS slope, $\beta$, by $0.20 \pm 0.09$. This result is in agreement with slope estimates derived from the data at other zenith angles (thin lines in the figures, the intervals of $E_{0}=10^{16}-10^{17} \mathrm{eV}$ for $50^{\circ}$ and $E_{0}>10^{17} \mathrm{eV}$ for $78^{\circ}$ ). Finally, data points at high muon densities and the largest zenith angle $\left(78^{\circ}\right)$, which correspond to primary energies around $10^{18} \mathrm{eV}$, lie near the upper edge of the calculation uncertainty band, and even somewhat exceed the expectations for a pure iron flux. 

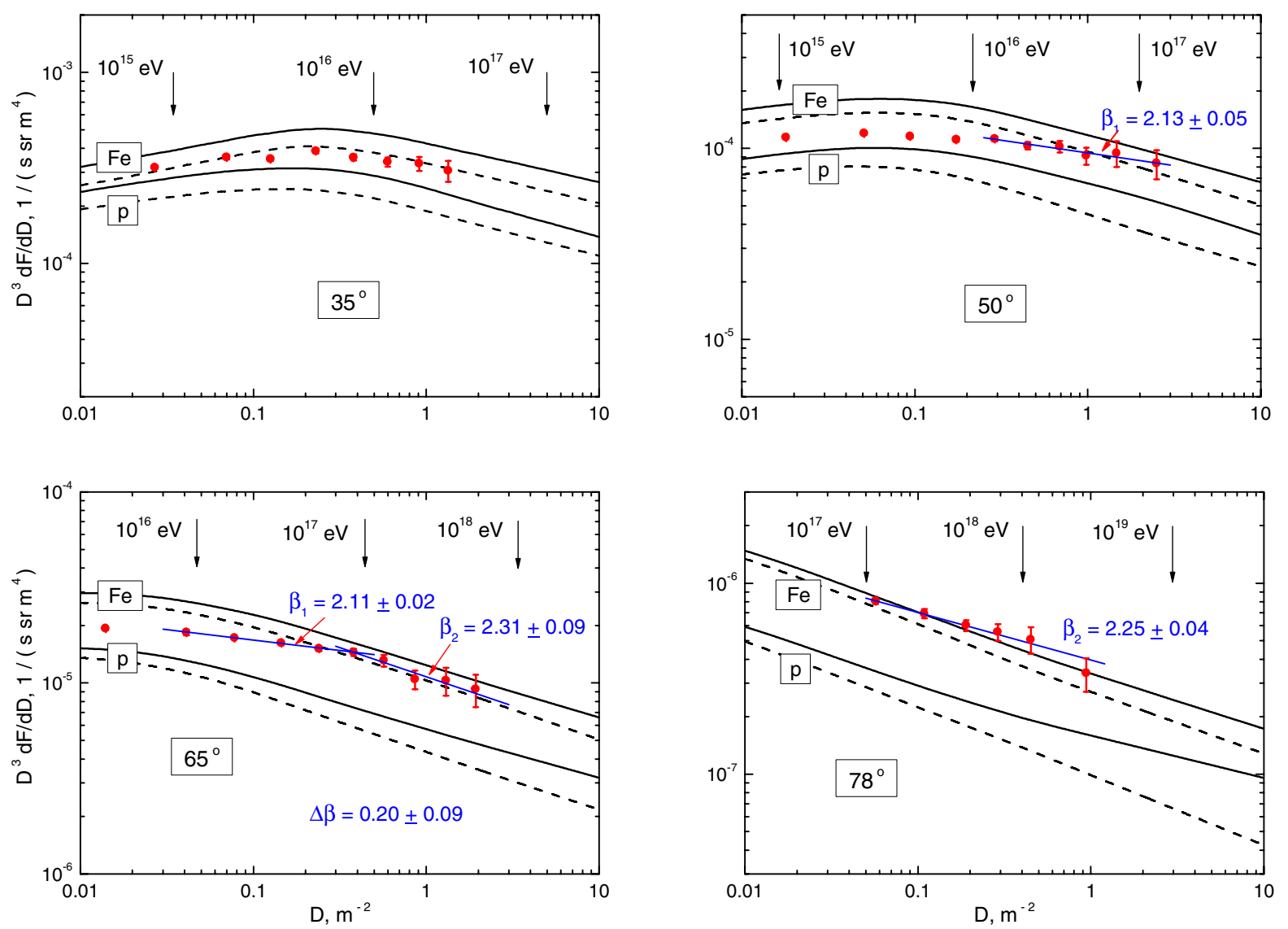

Figure 5. Measured (points) and calculated differential local muon density spectra for 4 zenith angles [7,10]. Thin lines represent partial power fits of the data between $10^{16}$ and $10^{17} \mathrm{eV}$ (integral spectrum slope $\beta_{1}$ ), and above $10^{17}$ primary energy $\left(\beta_{2}\right)$.

\section{Excess of muon bundles at ultra-high energies}

Under certain assumptions about primary mass composition and hadron interaction model, LMDS may be converted into estimates of the primary cosmic ray spectrum [7] and directly compared with other measurements of the primary energy spectrum. Such a procedure was applied to muon bundle samples with high muon multiplicities ( $m \geq 10$ ) and two zenith angle intervals: $\theta \geq 75^{\circ}$ and $\theta \geq 80^{\circ}$ (395 and 49 events, respectively). These data correspond to mean logarithmic primary energies around $10^{18} \mathrm{eV}$ and higher. The conversion was performed with two limiting assumptions on primary composition (pure protons and pure iron nuclei), using five different hadron interaction models available in CORSIKA: SIBYLL2.1, QGSJET01, QGSJET-II, EPOS1.61 and EPOS1.99. Thus, for each of the two data samples, 10 estimates of the differential primary energy spectrum were derived $[7,10]$.

In Fig. 6, primary intensity estimates obtained from DECOR data are compared with results of measurements of the PCR energy spectrum in the EeV energy range. Though a reasonable agreement in absolute normalization with AGASA data [11] is observed, it is seen from the figure that none of the examined interaction models allows us to match the data on muon bundles at large zenith angles with PCR spectrum measurements performed by means of the fluorescence method in HiRes [12], Auger [13] and Telescope Array [14] experiments, even for extremely heavy mass composition. This contradiction becomes even more significant, if we take into account that both
HiRes and Auger data on the $X_{\max }$ distribution of EAS favor a light (predominantly proton) primary composition near $10^{18} \mathrm{eV}[15,16]$. If one assumes only proton primaries, the observed intensity of muon bundles will be about 2-3 times higher than the expectation. Recently, based on the analysis of the surface detector data on the muon content in inclined EAS, the Auger collaboration came to the same conclusion that "none of the current shower models, neither for proton or iron primaries, are able to predict as many muons as are observed" [17]. Later, the inconsistence muon abundance in EAS at ultrahigh energies has got the name "muon problem" or "muon puzzle".

\section{Observation of the second knee in the EAS muon component}

As mentioned above, after the modernization of electronic systems of the Cherenkov water detector, the combined operation of NEVOD and DECOR was resumed in 2012. The main task of the experiment now being conducted is the measurement of the energy deposit of muon bundles in the NEVOD calorimeter [18]. Meanwhile, the available statistics of muon bundle events is continuously increasing, and by February 2016 the total net operation time of DECOR exceeded $41,000 \mathrm{~h}$. The differential local muon density spectra for three zenith angles $\left(57^{\circ}, 64^{\circ}\right.$ and $\left.72^{\circ}\right)$ reconstructed on the basis of the combined statistics of 2002-2007 and 2012-2016 (for 3 zenith angle intervals, 18187,17540 and 2920 bundles with muon multiplicity $m \geq 5$ ) are shown in Fig. 7. Arrows in the figure indicate 


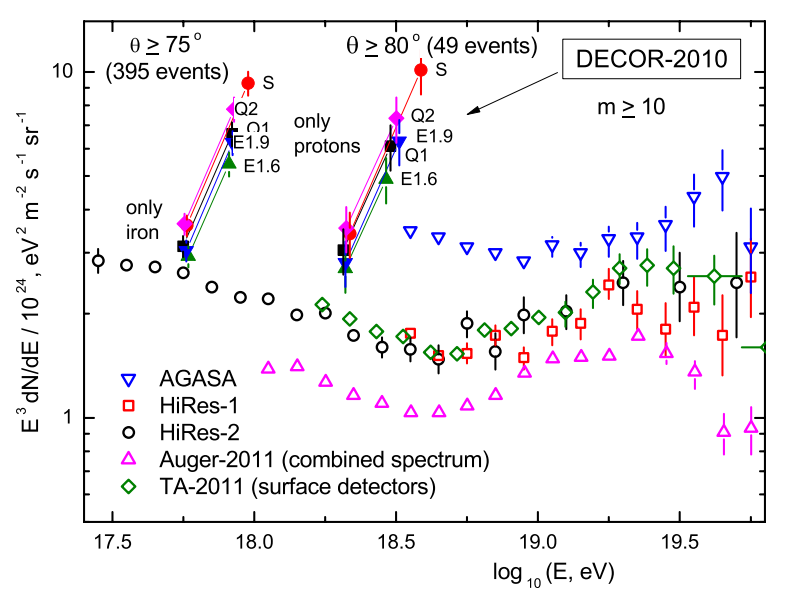

Figure 6. Comparison of the differential primary energy spectrum estimates derived from DECOR data on muon bundles (dark points) with the results of EAS measurements in AGASA [11], HiRes [12], Auger [13] and TA [14] experiments. Labels near the points indicate hadron interaction models mentioned in the text.

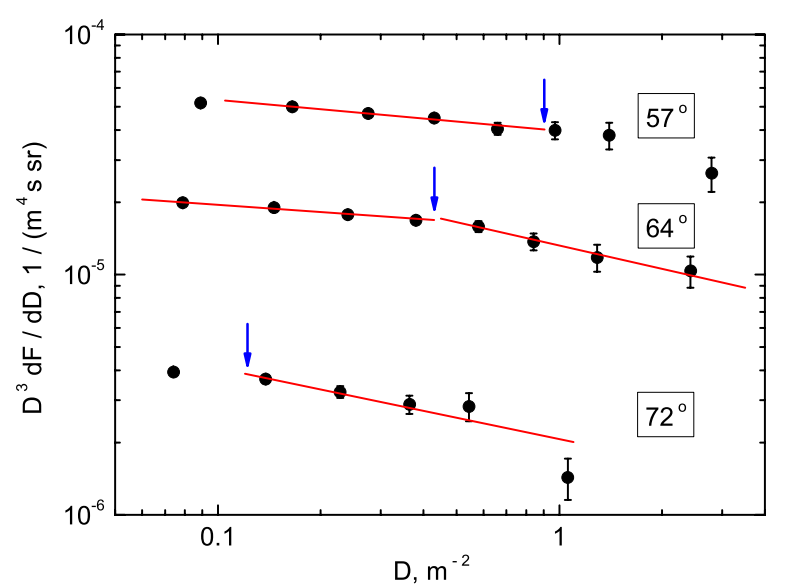

Figure 7. The LMDS reconstructed on the basis of combined DECOR data of 2002-2016 for three different zenith angles and piece-wise power function fits of the data (lines). Arrows indicate the position of the effective $10^{17} \mathrm{eV}$ primary particle energy for different angles.

the positions of the effective $10^{17} \mathrm{eV}$ primary particle energy for different zenith angles. Thin lines represent the power function fits of the spectra in two ranges of the primary energy: below and above $10^{17} \mathrm{eV}$. As seen from the figure, there are two well separated spectrum slopes in the two energy regions. Combining statistically independent results of the fits for different zenith angles, we obtain $\beta_{1}=2.107 \pm 0.014$ below $10^{17} \mathrm{eV}$ and $\beta_{2}=2.303 \pm 0.054$ above this energy, or $\Delta \beta=0.196 \pm$ 0.056 . This is the first statistically significant confirmation of the presence of the second knee measured with muons (at about $3.5 \sigma$ level).

\section{Conclusions}

During long-term measurements conducted with the NEVOD-DECOR experimental complex, unique data on inclined muon bundles detected at ground level have been accumulated. Application of a novel approach to
EAS studies based on the phenomenology of local muon density spectra at various zenith angles gave the possibility to obtain important information on the primary cosmic ray flux and interaction characteristics in the energy range from $10^{15}$ to $10^{18} \mathrm{eV}$ in the frame of a single experiment with a relatively small setup. Comparison of the measurement results with CORSIKAbased simulations showed the increase of the LMDS slope at $\mathrm{PeV}$ energies of primaries (corresponding to the first knee), and a trend to a heavier primary mass composition at energies above the knee. At large zenith angles and high muon densities (corresponding to primary energies around $10^{18} \mathrm{eV}$ ) a considerable excess of muon bundle events in comparison with the expectation based on independent estimates of the primary spectrum and widely used hadron interaction models has been found. The presence of the second knee (the steepening of the spectrum around $10^{17} \mathrm{eV}$ ) in multi-muon event intensity has been confirmed with $3.5 \sigma$ significance.

Experimental investigations are conducted at the Experimental Complex NEVOD (MEPhI) with support from the Ministry of Education and Science of the Russian Federation (contract RFMEFI59114X0002, MEPhI Academic Excellence Project, and government task). Simulations were performed using the resources of the MEPhI high-performance computing center.

\section{References}

[1] V.M. Aynutdinov et al., Astrophys. Space Sci. 258, 105 (1998)

[2] G. Battistoni et al., NIM A 245, 277 (1986)

[3] N.S. Barbashina et al., Instruments and Experim. Techniques 43, 743 (2000)

[4] N.S. Barbashina et al., Nucl. Phys. B (Proc. Suppl.) 165, 317 (2007)

[5] S.S. Khokhlov et al., Astrophys. Space Sci. Trans. 7, 271 (2011)

[6] N.S. Barbashina et al., Instruments and Experim. Techniques 51, 180 (2008)

[7] A.G. Bogdanov et al., Phys. Atom. Nucl. 73, 1852 (2010)

[8] D. Heck et al., Forschungszentrum Karlsruhe Report FZKA 6019 (1998)

[9] S. Eidelman et al. (Particle Data Group), Phys. Lett. B 592, 228 (2004)

[10] O. Saavedra et al., J. Phys.: Conf. Ser. 409, 012009 (2013)

[11] M. Takeda et al., Astropart. Phys. 19, 447 (2003)

[12] R.U. Abbasi et al., Phys. Rev. Lett. 100, 101101 (2008)

[13] J. Abraham et al., Phys. Lett. B 685, 239 (2010)

[14] D. Ikeda et al., Proc. 32nd ICRC (Beijing), vol. 2, 238 (2011)

[15] R.U. Abbasi et al., Phys. Rev. Lett. 104, 161101 (2010)

[16] J. Abraham et al., Phys. Rev. Lett. 104, 091101 (2010)

[17] G. Rodrigues et al., Proc. 32nd ICRC (Beijing), vol. 2, 95 (2011)

[18] A.G. Bogdanov et al., EPJ Web Conf. 99, 06004 (2015) 